\title{
Differences in Parotid Dosimetry and Expected Normal Tissue Complication Probabilities in Whole Brain Radiation Plans Covering C1 Versus C2
}

\author{
Andrew Orton ${ }^{1}$, John Gordon ${ }^{2}$, Tyler Vigh ${ }^{2}$, Allison Tonkin ${ }^{3}$, George Cannon ${ }^{2}$ \\ 1. Radiation Oncology, University of Utah Huntsman Cancer Hospital 2. Radiation Oncology, \\ Intermountain Medical Center 3. Radiology, Intermountain Medical Center
}

$\square$ Corresponding author: Andrew Orton, andrew.orton@hci.utah.edu

Disclosures can be found in Additional Information at the end of the article

\section{Abstract \\ Objectives}

There is no consensus standard regarding the placement of the inferior field border in whole brain radiation therapy (WBRT) plans, with most providers choosing to cover the first versus (vs.) second cervical vertebrae (C1 vs. C2). We hypothesize that extending coverage to C2 may increase predicted rates of xerostomia.

\section{Methods}

Fifteen patients underwent computed tomography (CT) simulation; two WBRT plans were then produced, one covering $\mathrm{C} 2$ and the other covering $\mathrm{C} 1$. The plans were otherwise standard, and patients were prescribed doses of 25, 30 and 37.5 gray (Gy). Dose-volume statistics were obtained and normal tissue complication probabilities (NTCPs) were estimated using the Lyman-Burman-Kutcher model. Mean parotid dose and predicted xerostomia rates were compared for plans covering C2 vs. C1 using a two-sided patient-matched t-test. Plans were also evaluated to determine whether extending the lower field border to cover C2 would result in a violation of commonly accepted dosimetric planning constraints.

\section{Results}

The mean dose to both parotid glands was significantly higher in WBRT plans covering C2 compared to plans covering $\mathrm{C} 1$ for all dose prescriptions $(\mathrm{p}<0.01)$. Normal tissue complication probabilities were also significantly higher when covering C2 vs. C1, for all prescribed doses $(\mathrm{p}<0.01)$. Predicted median rates of xerostomia ranged from $<0.03 \%-21 \%$ for plans covering $\mathrm{C} 2$

Received 10/26/2016 Review began 11/02/2016 Review ended 04/12/2017 Published 05/03/2017

CC Copyright 2017

Orton et al. This is an open access article distributed under the terms of the Creative Commons Attribution License CC-BY 3.0., which permits unrestricted use, distribution, and reproduction in any medium, provided the original author and source are credited. vs. $<0.001 \%-12 \%$ for patients treated with plans covering $\mathrm{C} 1(\mathrm{p}<0.01)$, dependent on the treatment dose and NTCP model. Plans covering C2 were unable to constrain at least one parotid to $<20 \mathrm{~Gy}$ in $31 \%$ of plans vs. $9 \%$ of plans when $\mathrm{C} 1$ was covered. A total parotid dose constraint of $<25$ Gy was violated in $13 \%$ of plans covering $\mathrm{C} 2 \mathrm{vs.} 0 \%$ of plans covering $\mathrm{C} 1$.

\section{Conclusions}

Coverage of C2 significantly increases the mean parotid dose and predicted NTCPs and results in more frequent violation of commonly accepted dosimetric planning constraints. 
Categories: Radiation Oncology

Keywords: xerostomia, palliation, dosimetry, ntcp, complications

\section{Introduction}

Whole brain radiation therapy (WBRT) is one of the most common treatments delivered in radiation oncology. Whole brain radiation therapy is typically utilized in the palliative setting when treating patients with multiple brain metastases. Less frequently, it is delivered in the curative setting, including as prophylactic cranial irradiation (PCI) for small-cell lung cancer and various high-risk or central nervous system (CNS) positive leukemias, as well as part of the definitive regimen in some institutions for primary CNS lymphomas. Several recent papers have proposed that the parotid glands be considered at-risk organs in patients receiving WBRT [1-2]. The poor prognosis traditionally associated with brain metastases previously limited interest in evaluating the long-term side effects of WBRT, as it was not expected that patients would live long enough to experience these late effects. However, the potential late effects of WBRT, including xerostomia, have become more prescient given the improved overall survival achieved in patients with brain metastases [3], as well as the potential for xerostomia to be induced during or shortly after radiation [4]. Additionally, it has been observed that the longterm impact of radiation on salivary function exists as a continuum and that doses as low as 1015 gray (Gy) may decrease parotid gland function [5]. In their review of publications that related dose-volume statistics to radiotherapy-induced xerostomia, Eisbruch, et al. noted that a lower mean dose to the parotid gland usually results in better salivary function, even at doses $<10$ Gy [6]. Specifically, they recommended keeping the dose to at least one gland <20 Gy. Another commonly accepted guideline to prevent xerostomia is to keep the mean dose to both parotids below $25 \mathrm{~Gy}$ [7]. When the mean dose to both parotids is limited to less than $25 \mathrm{~Gy}$, it has been estimated that salivary flow will return to baseline by 12 months [8]. Because xerostomia can be quite detrimental to the patient's quality of life, there has been interest in evaluating and subsequently limiting the dose to the parotid glands when delivering WBRT [9$11]$.

Whole brain radiation therapy is most commonly delivered using opposed lateral fields with 6 MV photons with gantry-rotation or multi-leaf collimators (MLCs) optimized to spare the lenses bilaterally. Even in the computed tomography (CT) era, radiation treatment fields continue to be designed based on anatomic landmarks identifiable on lateral radiographs of the skull, with a 5 to $10 \mathrm{~mm}$ margin placed on the cribriform plate and floor of the middle cranial fossa and 1 to $2 \mathrm{~cm}$ of "flash" beyond the anterior, superior, and posterior cranium to ensure dose homogeneity [12]. Variations in the WBRT technique can lead to substantial differences in the mean parotid dose [13]. The dose of radiation delivered incidentally to the parotid glands is influenced primarily by the location of the inferior border of the treatment field. Most commonly, the inferior border for the field is placed at either the inferior endplate of the $\mathrm{C} 1$ or $\mathrm{C} 2$ vertebra. The decision of which vertebral level to cover is typically a matter of physician preference, although coverage through $\mathrm{C} 2$ has most commonly been recommended when treating small-cell lung cancer and leukemia via the German Helmet technique [12,14]. The purpose of this study is to compare the dose delivered to the parotid glands in plans covering C1 versus C2. Normal tissue complication probabilities (NTCP) for these two approaches were then calculated and compared.

\section{Materials And Methods}

Fifteen patients underwent computed tomography (CT) simulation in our facility following immobilization with thermoplastic masks in the supine position. CT images were obtained from the vertex of the skull to the top of the thoracic spine at $2.5 \mathrm{~mm}$ intervals. The right and left parotid glands were contoured by a resident physician (AO) and the contours were subsequently reviewed independently by an attending radiation oncologist (GC) and an attending 


\section{Cureus}

neuroradiologist (AT), with discrepancies resolved by consensus (see Figure 1).

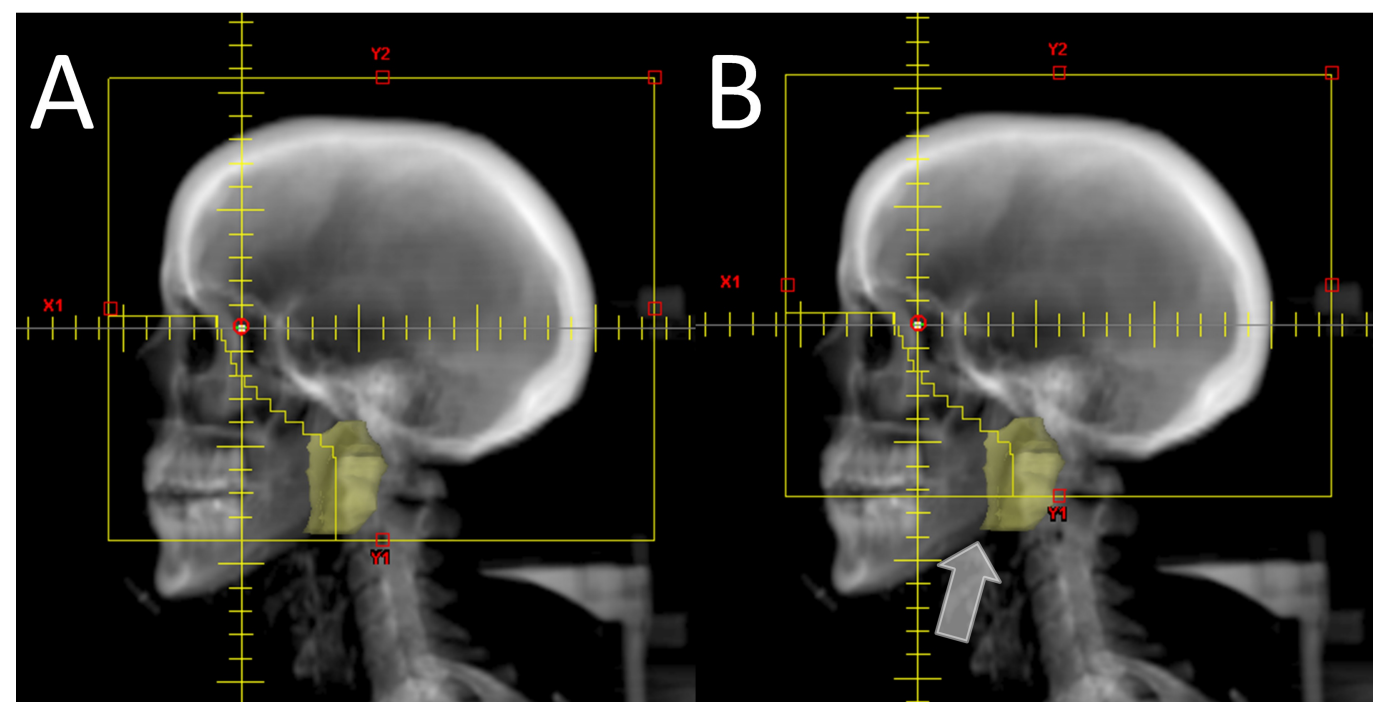

FIGURE 1: An example of differences in parotid coverage when the lower field is set at C1 vs. C2

An example of a WBRT plan with coverage of C2 (panel A) vs. C1 (panel B). The parotid volume is shown in yellow. The portion of the parotid blocked by the inferior primary jaw of the C1 plan is indicated by the white arrow.

Two treatment plans were produced for each patient using the CT simulation images. In each plan, treatment was delivered using $6 \mathrm{MV}$ photons in an opposed lateral configuration with the first plan extending the inferior field border to include all of $\mathrm{C}$, while the second plan set the inferior border to include all of C2. The WBRT plans were otherwise standard, with MLCs used to create a $1 \mathrm{~cm}$ field edge margin on the bony landmarks demarcating the inferior border of the cranial contents, and isocenters placed within the posterior orbit to create a quasi-half beam technique and limit divergence to the contralateral lens. Given the variations in the PCI and WBRT doses employed, plans were generated for prescription doses of $25 \mathrm{~Gy}, 30 \mathrm{~Gy}$ and 37.5 Gy.

Dose-volume statistics were obtained using the Varian Eclipse External Beam Planning System. The normal tissue complication probability (NTCP) was estimated in the same manner published by Noh et al.; the Lyman-Burman-Kutcher model was used with parameters obtained from studies published by Eisbruch [6], Emami [15] and Roesink [16]. Mean dose to the parotids and NTCPs were compared between the two groups, and statistical significance was determined using a patient-matched two-sided t-test. Plans were also evaluated to determine whether extending the lower field border to cover C2 would result in the violation of commonly accepted dosimetric planning constraints (limiting at least one parotid to a mean dose of $<20$ Gy, and limiting both parotids to <25 Gy).

\section{Results}

The mean dose to both parotid glands was significantly higher in WBRT plans covering C2 compared to plans limited to covering C1 for $25 \mathrm{~Gy}, 30 \mathrm{~Gy}$ and 37.5 Gy plans (see Figure 2, panel A). Dosimetric differences between C1 plans and C2 plans are given in Table 1 . The mean parotid dose was 15.3 Gy vs. $11.9 \mathrm{~Gy}$ ( $\mathrm{p}<0.01)$ for the $25 \mathrm{~Gy}$ plan; $18.3 \mathrm{~Gy}$ vs. $14.3 \mathrm{~Gy}(\mathrm{p}<0.01)$ for the 30 Gy plan; and 23.4 Gy vs. 18.5 Gy $(\mathrm{p}<0.01)$ for the 37.5 Gy plan. These trends were 
unchanged when evaluating the right and left parotid glands individually or when combined as a single dosimetric volume.
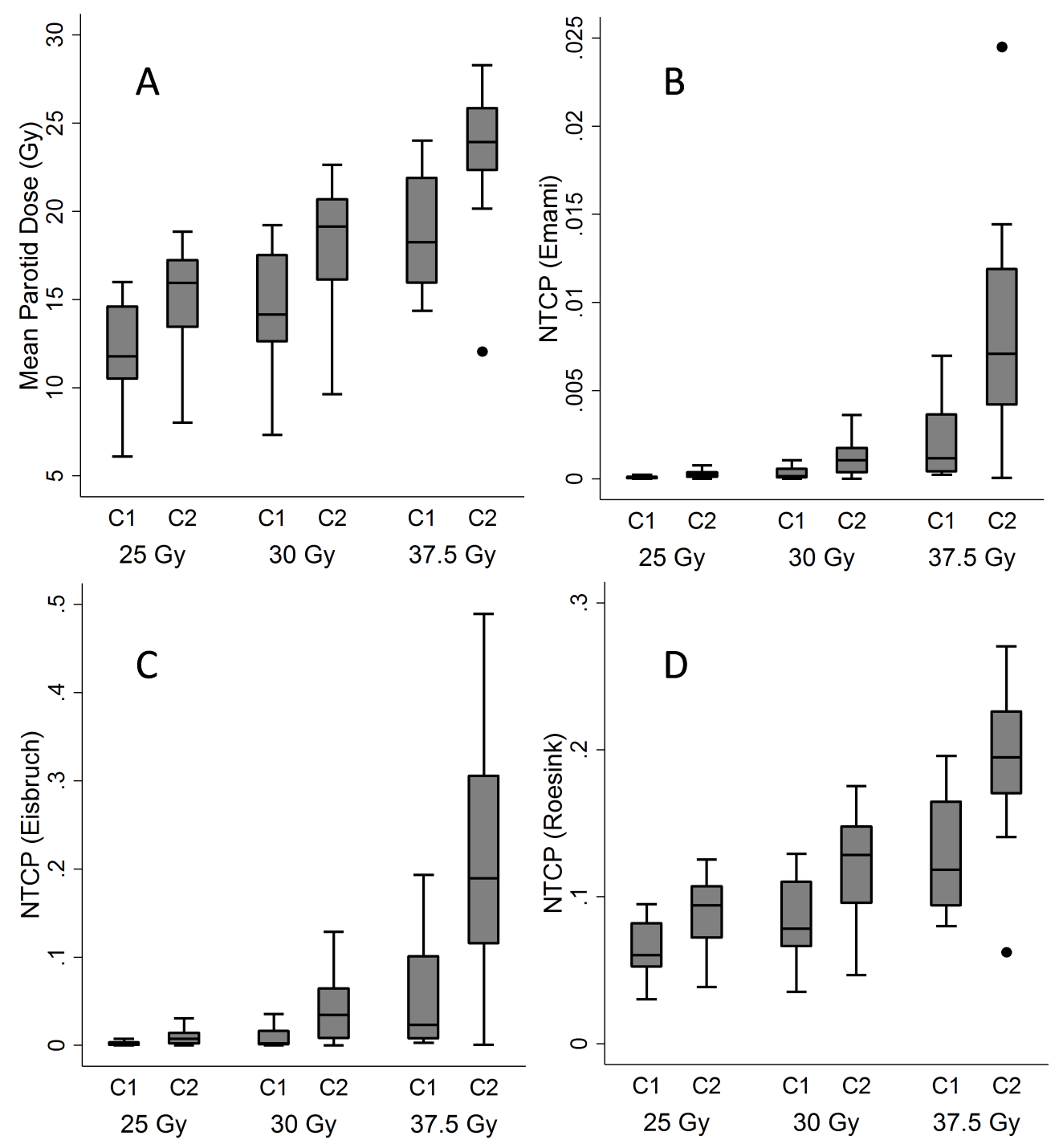

\section{FIGURE 2: A comparison of expected xerostomia rates}

Box and whisker plots representing the distribution of mean parotid dose (panel A), and NTCPs derived from parameters proposed by Emami, et al. (panel B), Eisbruch, et al. (panel C), and Roesink (panel D). Horizontal lines represent mean values for all 15 test patients, while the boxes span the 25 th percentile to 75 th percentile. Hashes denote 95 th percentiles and dots represent observations falling outside of the box and whisker distribution. 


\section{Cureus}

\begin{tabular}{|c|c|c|c|c|c|c|c|c|}
\hline & \multicolumn{2}{|l|}{25 Gy } & \multirow[t]{2}{*}{$\mathbf{p}$} & \multicolumn{2}{|l|}{$30 \mathrm{~Gy}$} & \multicolumn{2}{|c|}{37.5 Gy } & \multirow[b]{2}{*}{$p$} \\
\hline & C2 & C1 & & C2 & C1 & C2 & C1 & \\
\hline Mean parotid dose (Gy) & 15.3 & 11.9 & $<0.01$ & 18.3 & 14.3 & 23.4 & 18.5 & $<0.01$ \\
\hline V20 & $47.7 \%$ & $33.1 \%$ & $<0.01$ & $54.9 \%$ & $39.0 \%$ & $61.2 \%$ & $45.5 \%$ & $<0.01$ \\
\hline V25 & $6.8 \%$ & $3.0 \%$ & $<0.01$ & $45.3 \%$ & $31.3 \%$ & $56.2 \%$ & $40.9 \%$ & $<0.01$ \\
\hline NTCP (Emami) & $0.03 \%$ & $0.007 \%$ & $<0.01$ & $0.1 \%$ & $0.03 \%$ & $1.5 \%$ & $0.2 \%$ & $<0.01$ \\
\hline NTCP (Eisbruch) & $1.0 \%$ & $0.2 \%$ & $<0.01$ & $4.2 \%$ & $0.8 \%$ & $21.1 \%$ & $4.9 \%$ & $<0.01$ \\
\hline NTCP (Roesink) & $9.1 \%$ & $6.3 \%$ & $<0.01$ & $12.3 \%$ & $8.2 \%$ & $19.2 \%$ & $12.5 \%$ & $<0.01$ \\
\hline \multicolumn{9}{|c|}{ Violations of planning constraints } \\
\hline Total parotid dose <25 Gy & $0 / 15$ & $0 / 15$ & & $0 / 15$ & $0 / 15$ & $6 / 15$ & $0 / 15$ & \\
\hline At least one gland $<20 \mathrm{~Gy}$ & $0 / 15$ & 0/15 & & $3 / 15$ & 0/15 & $10 / 15$ & $4 / 15$ & \\
\hline
\end{tabular}

\section{TABLE 1: Differences in mean parotid dose, predicted NTCP rates, and dosiometric planning violations between plans covering $\mathrm{C} 2$ vs. $\mathrm{C} 1$}

NTCP: Normal tissue complication probabilities; V10: Volume of tissue receiving $20 \mathrm{~Gy}$; V25: Volume of tissue receiving 25 Gy.

Normal tissue complication probabilities for the parotid gland were also significantly higher when covering C2 vs. C1, for all prescribed doses (see Figure 2, panels B, C, and D). Mean predicted NTCPs for plans covering C2 vs. C1 are summarized in Table 1. Predicted rates of xerostomia, calculated using parameters proposed by Roesink et al. [16], ranged from 9\%-19\% vs. $6 \%-12 \%$ for patients treated with plans covering C2 vs. C1 $(\mathrm{p}<0.001)$. Estimates generated using the parameters suggested by Eisbruch, et al. [6] ranged from 1\%-21\% vs. $0.1 \%-5 \%$ in plans covering C2 vs. C1. Using the parameters suggested by Emami, et al. [15], we estimated the xerostomia risk to be very low at $<0.03 \%-1 \%$ vs. $>0.001 \%-0.2 \%$ for plans covering C2 vs. C1.

The effect of covering C2 vs. C1 on meeting commonly accepted planning constraints is summarized in Table 1. In our series, when the prescribed dose was 25 or $30 \mathrm{~Gy}$, we found that all patient plans limited to $\mathrm{C} 1$ were able to constrain at least one parotid to a mean dose <20 Gy. In contrast, $3 / 15$ (20\%) plans covering to C2 did not meet this criterion when 30 Gy was prescribed to cover $\mathrm{C} 2$. When the prescription dose was increased to $37.5 \mathrm{~Gy}, 4 / 15$ (27\%) of C1 plans and 10/15 (67\%) of C2 plans exceeded the constraint. Plans covering C1 and plans covering C2 were able to meet the total parotid dose constraint of $25 \mathrm{~Gy}$ when the dose prescription was 25 or $30 \mathrm{~Gy}$. When the prescription dose was increased to $37.5 \mathrm{~Gy}$, all plans covering C1 met this constraint, but 6/15 plans covering C2 did not.

\section{Discussion}

Xerostomia secondary to radiation-induced impairment of salivary flow can be a significant quality-of-life (QOL) issue for patients, resulting in dysphagia, dysgeusia, and increased risk of dental infections and caries. Salivary dysfunction is of particular concern in both the PCI and palliative WBRT settings. For patients cured following PCI for small-cell lung cancer and 
various high-risk or CNS-positive leukemias, chronic xerostomia can impact long-term QOL. The prospect of worsened QOL from xerostomia is also of special concern in the palliative setting as treatment toxicities must necessarily be balanced with the dual goals of life prolongation and maintenance of QOL.

The parotid glands are the dominant producers of serous saliva and are at risk for significant incidental radiation dose when delivering WBRT $[1,2,7,17]$. While historical data had suggested a salivary gland tolerance dose of $\sim 40 \mathrm{~Gy}$ [18], it is clear that parotid gland tolerance is in fact much lower than this. Eisbruch and colleagues reported in a seminal publication that a mean parotid dose of $26 \mathrm{~Gy}$ was associated with the threshold for impairment of stimulated salivary flow [6]. In this report, most glands that received over 26 Gy produced little saliva with no significant recovery through time. Partial volume thresholds were also reported by these investigators. Since the publication of this report, numerous other reports have characterized the dose-response relationship [18-19]. Results have varied regarding the predicted dose that would lead to xerostomia, but xerostomia with mean doses as low as $22 \mathrm{~Gy}$ has been identified [20].

Although data indicate recovery of salivary gland function with time, it can take up to 12 months to recover to pretreatment levels even when mean doses to the parotids are kept below 25 Gy [18]. Quantitative Analyses of Normal Tissue Effects in the Clinic (QUANTEC) guidelines therefore recommend sparing one parotid gland to a mean dose $<20 \mathrm{~Gy}$ if the paired gland is to receive a high dose or, if that is not possible, to limit the mean total bilateral parotid dose to $<25$ Gy $[5,19]$.

The radiation technique used to deliver WBRT can significantly affect the mean dose to the parotid glands due to their anatomic location $[10,13]$. There is historic concern that tightening the inferior borders of standard WBRT fields may result in underdosing the brain parenchyma [20-22]. Dosimetric studies have shown that in the 3-D planning era, the additional information provided by CT scans can limit dose to normal tissue while maintaining appropriate coverage of the intracranial space [11-13,23-25].

There is no set standard for the lower border of the WBRT field. Coverage to the bottom of the $\mathrm{C} 1$ or $\mathrm{C} 2$ vertebra originated in an era prior to cross-sectional imaging, with treatment to such osseous anatomic landmarks adhered to in order to avoid marginal tumor misses. The variable coverage of C1 vs. C2 seems to have been dictated by institutional preferences and practices rather than clinical data. Unfortunately, radiation-induced xerostomia is likely underreported in the palliative and prophylactic setting, leaving clinicians to depend on NTCP models to guide treatment planning decisions. We feel that the principle of primum non nocere should guide physicians to set the border at $\mathrm{C} 1$ unless radiographic or clinical disease characteristics indicate a high chance of failure at the inferior margin.

\section{Conclusions}

Extending the inferior border of a standard whole brain field to cover the $\mathrm{C} 2$ vertebral body significantly increases the mean parotid dose and the resultant anticipated rates of clinical xerostomia based on three separate NTCP models. Whole brain radiation therapy plans that cover $\mathrm{C} 2$ more often result in the violation of commonly accepted parotid treatment planning constraints compared to plans which are limited to covering $\mathrm{C} 1$. Clinicians who choose to prescribe dose to cover C2 in WBRT plans should be aware that doing so significantly increases the dose of radiation delivered to the parotid glands, and is predicted to result in more incidence of xerostomia at rates that may be as high as $20 \%$ in plans delivering $37.5 \mathrm{~Gy}$. Given the rarity of failures within the craniospinal axis at the $\mathrm{C} 2$ level, we would advocate against coverage of $\mathrm{C} 2$ in WBRT. 


\section{Additional Information}

\section{Disclosures}

Human subjects: All authors have confirmed that this study did not involve human participants or tissue. Animal subjects: All authors have confirmed that this study did not involve animal subjects or tissue. Conflicts of interest: In compliance with the ICMJE uniform disclosure form, all authors declare the following: Payment/services info: All authors have declared that no financial support was received from any organization for the submitted work. Financial relationships: All authors have declared that they have no financial relationships at present or within the previous three years with any organizations that might have an interest in the submitted work. Other relationships: All authors have declared that there are no other relationships or activities that could appear to have influenced the submitted work.

\section{References}

1. Noh OK, Chun M, Nam SS, Jang H, Jo S, Oh YT, Lim JC: Parotid gland as a risk organ in whole brain radiotherapy. Radiother Oncol. 2011, 98:223-226. 10.1016/j.radonc.2010.12.013

2. Fiorentino A, Chiumento C, Caivano R, Cozzolino M, Clemente S, Pedicini P, Fusco V: Whole brain radiotherapy: are parotid glands organs at risk?. Radiother Oncol. 2012, 103:130-131. 10.1016/j.radonc.2012.01.013

3. Kocher M, Soffietti R, Abacioglu U, et al.: Adjuvant whole-brain radiotherapy versus observation after radiosurgery or surgical resection of one to three cerebral metastases: results of the EORTC 22952-26001 study. J Clin Oncol. 2011, 29:134-141.

10.1200/JCO.2010.30.1655

4. Lal P, Bajpai R, Khurana R, et al.: Changes in salivary flow rates in head and neck cancer after chemoradiotherapy. J Cancer Res Ther. 2010, 6:458-462. 10.4103/0973-1482.77105

5. Deasy JO, Moiseenko V, Marks L, Chao KS, Nam J, Eisbruch A: Radiotherapy dose-volume effects on salivary gland function. Int J Radiat Oncol Biol Phys. 2010, 76:S58-63. 10.1016/j.ijrobp.2009.06.090

6. Eisbruch A, Ten Haken RK, Kim HM, Marsh LH, Ship JA: Dose, volume, and function relationships in parotid salivary glands following conformal and intensity-modulated irradiation of head and neck cancer. Int J Radiat Oncol Biol Phys. 1999, 45:577-587.

7. Milano MT, Marks LB, Constine LS: Survivorship and late effects. Clinical Radiation Oncology. Gunderson \& Tepper (ed): Elsevier, Philadelphia; 2012. 245-246.

8. Li J, Shan Z, Ou G, Liu X, Zhang C, Baum BJ, Wang S: Structural and functional characteristics of irradiation damage to parotid glands in the miniature pig. Int J Radiat Oncol Biol Phys. 2005, 65:1510-1516. 10.1016/j.ijrobp.2005.04.029

9. Loos G, Paulon R, Verrelle P, Lapeyre M: Whole brain radiotherapy for brain metastases: the technique of irradiation influences the dose to parotid glands. Cancer Radiother. 2012, 16:136-139. 10.1016/j.canrad.2011.11.002

10. Fiorentino A, Caivano R, Chiumento C, Cozzolino M, Pedicini P, Clemente S, Fusco V: Technique of whole brain radiotherapy: conformity index and parotid glands . Clin Oncol. 2012, 24:e140-141. 10.1016/j.clon.2012.07.003

11. Andic F, Ors Y, Niang U, Kuzhan A, Dirier A: Dosimetric comparison of conventional helmetfield whole-brain irradiation with three-dimensional conformal radiotherapy: dose homogeneity and retro-orbital area coverage. Br J Radiol. 2009, 82:118-122. 10.1259/bjr/37984200

12. Weiss E, Krebeck M, Kohler B, Pradier O, Hess CF: Does the standardized helmet technique lead to adequate coverage of the cribriform plate? An analysis of current practice with respect to the ICRU 50 report. Int J Radiat Oncol Biol Phys. 2001, 49:1475-1480.

13. Cho O, Chun M, Park SH, Oh YT, Kim MH, Park HJ, Nam SS, Heo J, Noh OK : Parotid gland sparing effect by computed tomography-based modified lower field margin in whole brain radiotherapy. Radiat Oncol J. 2013, 31:12-17. 10.3857/roj.2013.31.1.12

14. Le Pechoux C, Dunant A, Senan S, et al.: Standard-dose versus higher-dose prophylactic cranial irradiation (PCI) in patients with limited-stage small-cell lung cancer in complete remission after chemotherapy and thoracic radiotherapy (PCI 99-01, EORTC 22003-08004, 
RTOG 0212, and IFCT 99-01): a randomised clinical trial. Lancet Oncol. 2009, 10:467-474. 10.1016/S1470-2045(09)70101-9

15. Burman C, Kutcher GJ, Emami B, Goitein M: Fitting of normal tissue tolerance data to an analytic function. Int J Radiat Oncol Biol Phys. 1991, 21:123-135.

16. Roesink JM, Moerland MA, Battermann JJ, Hordijk GJ, Terhaard CH: Quantitative dose-volume response analysis of changes in parotid gland function after radiotherapy in the head-andneck region. Int J Radiat Oncol Biol Phys. 2001, 51:938-946.

17. King MT, Modlin L, Million L, Donaldson SS, Gibbs IC, Choi CY, Soltys SG: The Parotid Gland is an Underrecognized Organ at Risk for Craniospinal Irradiation. Technol Cancer Res Treat. 2015, 15:472-479. 10.1177/1533034615583406

18. Li Y, Taylor JM, Ten Haken RK, Eisbruch A: The impact of dose on parotid salivary recovery in head and neck cancer patients treated with radiation therapy. Int J Radiat Oncol Biol Phys. 2007, 67:660-669. 10.1016/j.ijrobp.2006.09.021

19. Moiseenko V, Wu J, Hovan A, Saleh Z, Apte A, Deasy JO, Harrow S, Rabuka C, Muggli A, Thompson A: Treatment planning constraints to avoid xerostomia in head-and-neck radiotherapy: an independent test of QUANTEC criteria using a prospectively collected dataset. Int J Radiat Oncol Biol Phys. 2012, 82:1108-1114. 10.1016/j.ijrobp.2011.04.020

20. Williams MV: The cribriform plate: a sanctuary site for meningeal leukaemia . Br J Radiol. 1987, 60:469-475. 10.1259/0007-1285-60-713-469

21. Carrie C, Hoffstetter S, Gomez F, et al.: Impact of targeting deviations on outcome in medulloblastoma: study of the French Society of Pediatric Oncology (SFOP). Int J Radiat Oncol Biol Phys. 1999, 45:435-439.

22. Miralbell R, Bleher A, Huguenin P, et al.: Pediatric medulloblastoma: radiation treatment technique and patterns of failure. Int J Radiat Oncol Biol Phys. 1997, 37:523-529.

23. Gripp S, Doeker R, Glag M, et al.: The role of CT simulation in whole-brain irradiation . Int J Radiat Oncol Biol Phys. 1999, 45:1081-1088.

24. Gripp S, Kambergs J, Wittkamp M, Get al.: Coverage of anterior fossa in whole-brain irradiation. Int J Radiat Oncol Biol Phys. 2004, 59:515-520. 10.1016/j.ijrobp.2003.10.030

25. Yu JB, Shiao SL, Knisely JP: A dosimetric evaluation of conventional helmet field irradiation versus two-field intensity-modulated radiotherapy technique. Int J Radiat Oncol Biol Phys. 2007, 68:621-631. 10.1016/j.ijrobp.2006.12.004 